febrile UTI with Escherichia Coli occurred two weeks later and was treated successfully with ten-day course of Ciprofloxacin.

Ultrasound examination revealed normal kidneys and bladder, while voiding cystography was evocative for bilateral VUR and post void residual.

Neurologic status improved over a three-month period from almost complete hypotonic palsy to mild hypotonic paraplegia.

Discussions Long hospitalization and prolonged urine catheterization increase the risk of CAUTI. High dose Meropenem remains efficient in treating MDR Klebsiella strains. Recurrent UTI should be further investigated for presence of VUR.

Neurological damage, like hypotonic cerebral palsy can be associated with underactive bladder and detrusor underactivity.

\section{A NOVEL COL4A4 MUTATION IN THE PROBAND INITIALLY DIAGNOSED AS IGAN WITH AUTOSOMAL RECESSIVE ALPORT SYNDROME}

Ilayda Altun*, Seha Saygılı, Nur Canpolat, Salim Çalışkan, Lale Sever. Istanbul UniversityCerrahpasa, Division of Pediatric Nephrology, Istanbul, Turkey

\subsection{6/archdischild-2021-europaediatrics.359}

Immunoglobulin A nephropathy (IgAN) is recognized as the most common form of glomerulonephritis all over the world. The diagnosis of IgAN is generally made according to clinical findings and histologic lesions on renal biopsy. Alport Syndrome is an inherited rare glomerular disorder characterized by hematuria, proteinuria, sensorineural hearing loss and progressive kidney disease. We here present a patient initially diagnosed as IgAN with biopsy findings and then diagnosed as Alport Syndrome by genetic screening.

An eight-year-old boy presented with a history of macroscopic hematuria attacks preceded by upper respiratory tract infections. Renal biopsy was compatible with IgAN showing positive staining of $\operatorname{IgA}$ in the mesangium and the basement membrane in immunofluorescence. Furthermore, the thinning and thickened of the glomerular basement membrane and splitting of lamina densa was found in electron microscopic examination. During his follow-up, persistent microscopic hematuria and proteinuria were noticed.

Sensorineural hearing loss was developed at ten years of age. Also the proband had a family history of hematuria with proteinuria. Despite the positive mesangial IgA staining in immunofluorescence, atypical renal phenotypes for IgAN including persistent hematuria and hearing loss, and positive family history and electron microscopic findings of renal biopsy aroused suspicion of Alport Syndrome. Genetic analysis (whole exon sequencing) demonstrated a homozygous mutation in COL4A4 (chr 2:g.227922261 c.2438delG (p. Gly813AspfsTer56) in the proband. Heterozygous mutation was identified by Sanger sequencing of gene COL4A4 in the carrier of all member of his family.

Due to this very rare coincidence, we emphasize that atypical clinical findings should warn the clinicians for other possible diagnosis. The diagnosis of this case also highlights the importance of genetic test in diagnosis of inherited kidney disease. Genetic screening has been recommended as the gold standard for the proper clinical diagnosis and understanding the mode of inheritance when Alport syndrome has suspected in a patient.

\section{0} UNILATERAL RENAL AGENESIA : A 28-YEAR SINGLE CENTER EXPIRIENCE

Dragan Kosic*, Srdjan Danojlic. OB Studenica Kraljevo, Radiology department

10.1136/archdischild-2021-europaediatrics.360

Unilateral renal agenesis (URA) is defined as the one-sided congenital absence of renal tissue resulting from failure of embryonic kidney formation [1] URA is often associated with congenital anomalies of the kidney and urinary tract and extra-renal anomalies, such as cardiac, genital or gastrointestinal malformations. The impaired clinical outcome of those children may be explained by the hyperfiltration hypothesis which implies that children with a solitary functioning kidney are at risk to develop hypertension, proteinuria, and chronic kidney disease. [2] Aim of this paper was to estimate the incidence of URA and associated anomalies in our population.

We reviewed 44 consecutive cases of unilateral renal agenesis diagnosed at our hospital in this 28 years (1991-2019) retrospective study During that period 28776 children were evaluated by 56921 renal ultrasonography (RUS). Patient age at diagnosis ranged from newborn to 18 years. There were 23 $(52 \%)$ boys and $21(48 \%)$ girls. Our patients were evaluated for urinary tract infection or abdominal pain and during examination for congenital malformations or pathological fetal US. The left kidney was absent in $25(56 \%)$ patients and the right kidney was absent in the remaining 19 (44\%). Associated genital anomalies were present in 5 (23\%) of 21 girls with URA including agensis et hypoplasio uteri, agenesio et polycystic ovarii, vaginal duplicity. One boy (4\%) had contralateral hydronephrosis with bladder diverticulum. Ectopic kidney was diagnosed in 15 patients.

In our population the frequency of URA was 1 per 1330 births. The most frequent non urinary malformations were genital anomalies among girls.

Careful screening should be proposed throughout childhood to detect early signs of glomerular hyperfiltration and prevent its progression to more serious complications. Ultrasound has been effective for early detection of renal and urinary tract anomalies.

\section{PRETREATMENT MORNING URINE OSMOLALITY PREDICTS ORAL DESMOPRESSIN LYOPHILISATE TREATMENT OUTCOME IN PATIENTS WITH PRIMARY MONOSYMPTOMATIC ENURESIS}

Iva Hižar, Martin Ćuk, Milan Milošević, Antonella Geljić, Marijan Saraga, Slaven Abdović* Department of Pediatric Nephrology, Children's Hospital Zagreb, Croatia

\subsection{6/archdischild-2021-europaediatrics.361}

To determine the association between urine osmolality in patients with primary monosymptomatic enuresis (PMNE) and their response to desmopressin.

We hypothesized that pretreatment morning urine osmolality is lower in PMNE patients with complete response to desmopressin treatment compared to the cases with partial or noresponse.

This was a prospective cohort study that included 419 patients with enuresis seen in our outpatient clinic between October 2017 and October 2019. Patient workup included symptom checklist, bladder diary, kidney and bladder 
ultrasound scan, uroflow, urinalysis and culture, urine $\mathrm{Ca} / \mathrm{crea}-$ tinine, and first-morning urine osmolality. Patients $<5$ years of age, with secondary enuresis, and those who did not show at the follow-up visit were excluded.

Oral desmopressin lyophilisate was recommended to all patients with PMNE and normal bladder capacity. After one month of therapy, initial success was assessed according to ICCS. Correlation coefficients were used to identify variables that were significantly correlated to complete response. ROC analysis was used to determine the urine osmolality cut-off value. Odds ratio and correlation coefficients in favor of complete initial success were analyzed with binary logistic regression.

There were 48 patients with PMNE who received desmopressin and were followed for treatment success. Of tested variables, only lower urine osmolality was found to be significantly in favor of complete response to desmopressin therapy. ROC analysis determined the value of $\leq 814 \mathrm{mOsm} / \mathrm{L}$ as a cut-off value for complete success (sensitivity 65\% and specificity $75 \%$ ). The odds ratio for complete success with desmopressin therapy in PMNE patients with first-morning urine osmolality $\leq 814 \quad \mathrm{mOsm} / \mathrm{L}$ was $9.086 \quad(95 \%$ CI $1.893 \quad$ 43.618, $\mathrm{P}=0.006$ ).

For PMNE patients with high pretreatment morning urine osmolality, an alternative treatment to desmopressin should be considered because of the significantly higher risk of treatment failure.

\section{\begin{tabular}{l|l}
362 & 3D PRINTED PEEK EXTRAVASCULAR STENT IN
\end{tabular} TREATMENT OF NUTCRACKER SYNDROME IN AN ADOLESCENT BOY}

Da-li He, Heng-en Wang, Yong Jiao, Bin Wu, Dong Cui, Yan-yan Li, Yong Yang, Tiesheng Cao, Danko Milosevic*, Bo Zhang. University Hospital Centre Zagreb

10.1136/archdischild-2021-europaediatrics.362

A 14-year-old boy with constant and intense periumbilical pain for the past 24 months caused by nutcracker syndrome (NCS) (visual analog pain scale, 8-9) was treated with 3D printed PEEK extravascular stent. To our best knowledge this is the first European application in a child. 3D model reconstruction showed that the left renal vein (LRV) and the duodenum were contracted at almost the same level via aortomesenteric angle, resulting in LRV compression from the abnormal high-level duodenal compartment. Surgical procedure included a dissection of fibrous bundles which freed the duodenum. LRV was dissected out medially from the renal hilum to the vena cava inferior until it was completely mobilized. A complete resection off the narrow fibrous ring between abdominal aorta and superior mesenteric artery was necessary. 3D printed PEEK extravascular stent via camera port was placed and bound to the narrowed portion of LRV to prevent blood vessel compression. Such stent demonstrates significant advantages over open or other minimally invasive surgery such as endovascular stenting and artificial vessels procedures with expanded polytetrafluoroethylene. It has distinctive personalized design and good rigidity as well as durability which allow vascular growth, preventing stent migration and thrombosis as well. Therefore, it is suitable for both adult and pediatric patients.

\section{TWO BOYS WITH C3/DDD GLOMERULONEPHRITIS}

Danko Milosevic*. University Hospital Centre Zagreb

10.1136/archdischild-2021-europaediatrics.363

A boy, 16 years of age. During routine analysis hematuria and proteinuria (up to $1.84 \mathrm{~g} / \mathrm{L}$ ) were found along with hypertension ( $\max 160 / 70 \mathrm{mmHg}$ ). Physical examination showed no abnormailities. Total complement activity (classical pathway) of $7 \mathrm{CH} 50 / \mathrm{ml}$ (ref. 48-103 $\mathrm{CH} 50 / \mathrm{ml}$ ) and total complement activity (alternative pathway) of 0\% (ref. 70-105\%).

C3 levels were low (median $0.06 \mathrm{~g} / \mathrm{L}$ ) while C4 were within reference ranges.

Anti-C1q IgG autoantibody was $677 \mathrm{U} / \mathrm{ml}$ (ref. <52), C3nephritic factor $11,0 \%$ (ref. $<10 \%$ ) and sC5b-9 (terminal complement complex) $1640 \mathrm{ng} / \mathrm{mL}$ (ref. 110-252 ng/mL). Complement factor I antigen was decreased 51\% (ref. 70$130 \%$ ) as well as complement factor B antigen of $45 \%$ (ref. $70-130 \%)$.

These results support the presence of AP dysregulation with overactivation.

Lupus aDNA negative Kidney biopsy revealed C3 glomerulonephritis. Due to nephritic syndrome the boy was treated with pulse methylprednisolone therapy, rituximab and cyclophosphamide. Until present, proteinuria was reduced (up to $0.48 \mathrm{~g} / \mathrm{L}$ ) with microhematuria. Due to a persistently low C3 and elevated sC5b-9, we are now considering Eculisumab as off label treatment option. Genetic analysis ongoing.

Second boy, 5 years of age, was treated for nephritic syndrome in regional hospital. Physical examination showed no abnormailities. As significant proteinuria $(3.62 \mathrm{~g} / \mathrm{L})$ with low C3 was found. A kidney biopsy revealed Dense deposit disease. Total complement activity (classical pathway) $18 \mathrm{CH} 50 /$ $\mathrm{ml}$ (ref. 48-103 $\mathrm{CH} 50 / \mathrm{ml}$ ) and total complement activity (alternative pathway) 0\% (ref. 70-105\%). C3 and C4 levels were low $(0.1$ and $0.06 \mathrm{~g} / \mathrm{L}$ respectively). C3-nephritic factor was elevated $41,3 \%$ (ref. $<10 \%$ ) as well as sC5b-9 (terminal complement complex) of $342 \mathrm{ng} / \mathrm{mL}$ (ref. 110-252 ng/mL).

$\mathrm{LDH}$ was elevated of $552 \mathrm{U} / \mathrm{L}$. Creatinine level was elevated at its highest of $198 \mu \mathrm{mol} / \mathrm{L}$. Activity of the alternative and classical pathways were both deficient. Complement C3 and C4 levels are decreased, while those of factor I and B are in the normal range. The terminal pathway activity marker level is increased. The above results support a complement activation and consumption involving at least the classical pathway. The boy was treated with pulse methylprednisolone therapy, rituximab and cyclophosphamide. After initial therapy elevated serum creatinine levels decreased, as well as proteinuria. C3 stabilized around $0.50, \mathrm{C} 4$ normalizes at 0.40 , while proteinuria decreased to $0.9 \mathrm{~g} / \mathrm{L}$.

\section{URINARY INCONTINENCE}

1,2,3 L Szabó* ${ }^{2}$ Bajusz, ${ }^{2} \mathrm{~K}$ Losonczi, ${ }^{2} \mathrm{~Gy}$ Réti, ${ }^{2} \mathrm{~B}$ Lombay, ${ }^{1} \mathrm{M}$ Polovitzer, ${ }^{1} \mathrm{D}$ Molnár, ${ }^{1} B$ Sulya, ${ }^{1} \mathrm{M}$ Sajthy, ${ }^{1} \mathrm{M}$ Merksz, ${ }^{1} \mathrm{~A}$ Kiss. ${ }^{1}$ Heim Pál National Paediatric Institute, Budapest; ${ }^{2}$ Velkey László Child Health Centre, Borsod County Central Hospital and University Teaching Hospital, Miskolc; ${ }^{3}$ Department of Family Care Methodology, Institute of Health Science, Semmelweis University, Budapest

10.1136/archdischild-2021-europaediatrics.364

Functional and morphological anomalies could cause urinary incontinence. The essence of urinary incontinence and 